\title{
Gaze-Centered Updating of Visual Space in Human Parietal Cortex
}

\author{
W. Pieter Medendorp, ${ }^{1,2,3,6}$ Herbert C. Goltz, ${ }^{1,4}$ Tutis Vilis, ${ }^{1,5}$ and J. Douglas Crawford ${ }^{1,2,3}$ \\ ${ }^{1}$ Canadian Institutes of Health Research Group on Action and Perception, ${ }^{2}$ Centre for Vision Research, and ${ }^{3}$ Department of Psychology, York University, \\ Toronto, Ontario, Canada, M3 1P3, Departments of ${ }^{4}$ Psychology and 5 Physiology and Pharmacology, University of Western Ontario, London, Ontario, \\ Canada, N6A 5C1, and ${ }^{6}$ F. C. Donders Centre for Cognitive Neuroimaging and Nijmegen Institute for Cognition and Information, University of Nijmegen, \\ NL 6500 HE, Nijmegen, The Netherlands
}

Single-unit recordings have identified a region in the posterior parietal cortex (PPC) of the monkey that represents and updates visual space in a gaze-centered frame. Here, using event-related functional magnetic resonance imaging, we identified an analogous bilateral region in the human PPC that shows contralateral topography for memory-guided eye movements and arm movements. Furthermore, when eye movements reversed the remembered horizontal target location relative to the gaze fixation point, this PPC region exchanged activity across the two cortical lobules. This shows that the human PPC dynamically updates the spatial goals for action in a gaze-centered frame.

Key words: fMRI; remapping; spatial perception; arm; eye; human; spatial memory; spatial updating; parietal cortex; LIP; PRR

\section{Introduction}

The posterior parietal cortex (PPC) is important for spatial processing and visually guided action (Goodale and Milner, 1992; Jeannerod et al., 1995; Andersen et al., 1997; Colby and Goldberg, 1999). In the monkey, different regions within the PPC process information for different actions. For example, the lateral intraparietal sulcus (LIP) codes target location for eye movements called saccades (Gnadt and Andersen, 1988; Barash et al., 1991; Duhamel et al. 1992a; Colby et al., 1996; Mazzoni et al., 1996), whereas the adjacent parietal reach region (PRR) codes the same for impending reaching movements (Galletti et al., 1997; Snyder et al., 1997; Batista et al., 1999).

Both areas have been shown to encode this information explicitly in a gaze-centered, eye-fixed frame of reference, which must be updated across eye movements to remain accurate (Duhamel et al., 1992a; Batista et al., 1999) as opposed to a head or body-centered frame, which would be independent of eye movements (Scherberger et al., 2003). It is also thought that eye position, head position, and vestibular signals in these regions may be used to transform this gaze-centered information into other frames of reference (Andersen et al., 1985; Brotchie et al., 1995; Snyder et al., 1998).

Human neuroimaging studies have also implicated the PPC in saccades and arm movements (for review, see Corbetta et al.,

\footnotetext{
Received Feb. 25, 2003; revised March 13, 2003; accepted March 15, 2003.

This work was supported by grants from the Canadian Institutes of Health Research and the Canadian Natural Sciences and Engineering Research Council. W.P.M. and H.C.G. are supported by the Human Frontier Science Program. J.D.C. is supported by the Canada Research Chair Program. We thank Drs. M. A. Goodale, D. B. Tweed, and J.C. Culham for comments on this manuscript.

Correspondence should be addressed to Dr. W. P. Medendorp, F. C. Donders Centre for Cognitive Neuroimaging and Nijmegen Institute for Cognition and Information, University of Nijmegen, P.O. Box 9104, NL-6500 HE, Nijmegen, The Netherlands. E-mail: p.medendorp@nici.kun.nl.

Copyright $\odot 2003$ Society for Neuroscience $\quad 0270-6474 / 03 / 236209-06 \$ 15.00 / 0$
}

1998; Connolly et al., 2000; DeSouza et al., 2000; Culham and Kanwisher, 2001; Sereno et al., 2001), with a topographic organization related to different directions of target location (Sereno et al., 2001). Without varying eye position, however, it is unclear whether this topography is related to a gaze-centered frame of reference, let alone whether the human PPC shows spatial updating across eye movements. Here we performed two functional magnetic resonance imaging ( $\mathrm{fMRI}$ ) experiments to investigate whether the human PPC demonstrates gaze-centered coding and updating of spatial information for saccades and pointing movements.

\section{Materials and Methods}

MRI scanning and data analysis. Data were collected with a 4.0 Tesla Varian Siemens whole-body imaging system. Six male subjects (aged 21-35 years) viewed stimuli that were backprojected, using an NEC VT540 LCD projector (refresh rate, $70 \mathrm{~Hz}$ ) with custom optics, onto the ceiling of the magnet bore. All subjects but one were right-handed, and all pointing movements were made using the right hand. During experiment 1,19 contiguous slices were used to image the entire parietal cortex using a quadrature radio-frequency surface coil centered on the posterior parietal lobe. Functional data were obtained using navigator echo corrected $\mathrm{T} 2{ }^{\star}$-weighted segmented gradient echoplanar imaging [echo time (TE), $15 \mathrm{msec}$; flip angle (FA), $45^{\circ}$; field of view (FOV), $19.2 \times 19.2 \mathrm{~cm}$; repetition time (TR), $2 \mathrm{sec}$; in-plane pixel size, $3 \times 3 \mathrm{~mm}$; thickness, 4 $\mathrm{mm}]$. During experiment 2, five of the initial 19 slices, which included the region of interest identified in experiment 1 , were scanned at a higher temporal resolution $\left(\mathrm{FA}, 22^{\circ}\right.$; TR, $\left.0.5 \mathrm{sec}\right)$. Functional data were superimposed on high-resolution inversion prepared three-dimensional T1weighted anatomical images of the brain (typically 128 slices; $256 \times 256$; FOV, $19.2 \times 19.2 \mathrm{~cm}$; TE, $5.5 \mathrm{msec}$; TR, $10.0 \mathrm{msec}$ ) using a phase reference image that corrected for high-field geometric distortions. In separate sessions, subjects were rescanned using a birdcage-style head coil to obtain full brain anatomical images. A high-resolution inversion prepared three-dimensional T1-weighted sequence was used (FA, 15 ; voxel 
size, $1.0 \mathrm{~mm}$ in-plane; $256 \times 256$; 164 slices; TR, $0.76 \mathrm{sec}$; TE, $5.3 \mathrm{msec}$ ). Analysis was performed using Brain Voyager 4.6 software (Brain Innovation, Maastricht, The Netherlands) and Matlab software (MathWorks, Natick, MA). Surface coil images were realigned manually to head-coil images. Anatomical images for each subject were segmented at the gray-white matter boundary, rendered and inflated for visualization purposes only. For functional data analysis, we excluded any scans in which motion artifacts were observed. Time courses within each voxel were corrected for linear drift. Anatomical and functional images were transformed to Talairach space to obtain coordinates for the regions of interest.

Experiment 1: delayed-movement task. Subjects fixated a central letter, S, P, or F, referring to a delayed-saccade task (S), a delayed pointing task $(\mathrm{P})$, or a fixation task $(\mathrm{F})$, respectively (see Fig. 1A) (Snyder et al., 1997; Batista et al., 1999; Sereno et al., 2001). Then, a brief peripheral dot was presented for 250 msec, to either the left or right at random horizontal eccentricities from the continuous interval between 10 to $25^{\circ}$. Subsequently, a band of distractors $\left(70^{\circ}\right.$ horizontal $\times 8^{\circ}$ vertical; eccentricity of the dot, $0.8^{\circ}$; density, 0.14 dots per square degree) blinked (at $5 \mathrm{~Hz}$ ) for $2.5 \mathrm{sec}$, during which the subjects maintained central fixation (and pointed to the central letter $\mathrm{P}$ when in pointing mode). Then, at distractor offset, subjects made either a saccade or a pointing movement to the remembered target location and immediately back to center. Subjects made no movement in the fixation (F) task. During the pointing task, they were instructed to maintain central fixation of the eyes at all times. Pointing movements consisted of wrist rotations, such that the index finger pointed to the remembered target location (DeSouza et al., 2000). The subject's view of the hand was occluded with black cardboard during pointing (DeSouza et al., 2000). The time between successive movements was $5 \mathrm{sec}$.

Scans to determine the movement-related activation maps comprised 17 blocks (each $20 \mathrm{sec}$ ), in which saccade and pointing blocks (each including movements to four different target locations) were alternated with fixation blocks. Scans for topography comprised 12 blocks (each 20 sec), in which four leftward targets were alternated with four rightward targets. Typically, three scans for each task were obtained, which were averaged to improve the signal-to-noise level. A general linear model analysis was then used to determine activated and topographic voxels in the parietal and occipital cortex. We used a higher statistical threshold to test between movement activation and fixation $\left(p<10^{-5}\right)$ than for topography $(p<0.001)$.

Experiment 2: the intervening saccade task. The intervening saccade task used the classical Hallett and Lightstone (1976) double-step paradigm to investigate how spatial information for saccades and pointing is stored and updated during eye movements. As shown in Figure $1 B$, subjects fixated centrally, and two brief peripheral dots, a green (the goal target) and red dot (refixation target for the first saccade), were flashed (duration of $250 \mathrm{msec}$ ) after 0.5 and $1.0 \mathrm{sec}$, respectively. Both targets were either left or right of central fixation, at different random eccentricities, i.e., the red at $16-20^{\circ}$ from central fixation and the green target at $7-10^{\circ}$ on either side of the goal target. Subsequently, the horizontal band of distractors blinked. Then, $6 \mathrm{sec}$ after the start of the flashing pattern, the central fixation was switched off, signaling a saccade toward the remembered location of the red target. Then, after an additional $12 \mathrm{sec}$, the flashing distractors were turned off, and subjects made either a saccade or a pointing movement to the remembered location of the goal target. The paradigm had four different conditions (see Fig. 3A). The location of the remembered goal target after the intervening first saccade remained in the right hemifield (RR) or remained in the left (LL), or it moved from the left to the right hemifield (LR), or vice versa (RL). This test was designed to discriminate between gaze-centered updating of signals as opposed to coding in a saccade-independent frame such as head-, body-, or world-centered coordinate frames.

Each scan contained 12 epochs ( $25 \mathrm{sec}$ each), in which the four conditions were pseudorandomly interleaved. Three to four scans were run for both the saccade-saccade and the saccade-point task. For each scan, the mean fMRI signal at volumes $6-8$ across all 12 epochs was taken as baseline. Data was temporally smoothed using a moving average filter with a span of 5 (volumes). For additional data analysis, for each condition, a mean signal and SD were computed at each volume. Furthermore, when differences between conditions were computed, uncertainties were determined using standard statistical rules for uncertainty combination.

Movement recordings. Each subject extensively practiced all tasks before imaging to ensure that these were performed correctly. In addition, eye movement recordings (Applied Science Laboratories, Bedford, MA) were performed on three of our subjects outside the scanner for the saccade-saccade and the saccade-point task to confirm that they followed the instructions correctly. This also confirmed that subjects were able to keep fixation while making pointing movements. Moreover, the fMRI experiment was self-controlling for eye movements: the pseudorandom interleaving of the conditions was designed so that positive results (like those we observed) would only be obtained if subjects made eye movements to the correct locations. Pointing movements were not recorded. However, in our fMRI analysis, we focused on brain activity in the delay periods before the pointing movements, so any small errors made during the subsequent movement would likely be reflected in brain events that we did not record. 


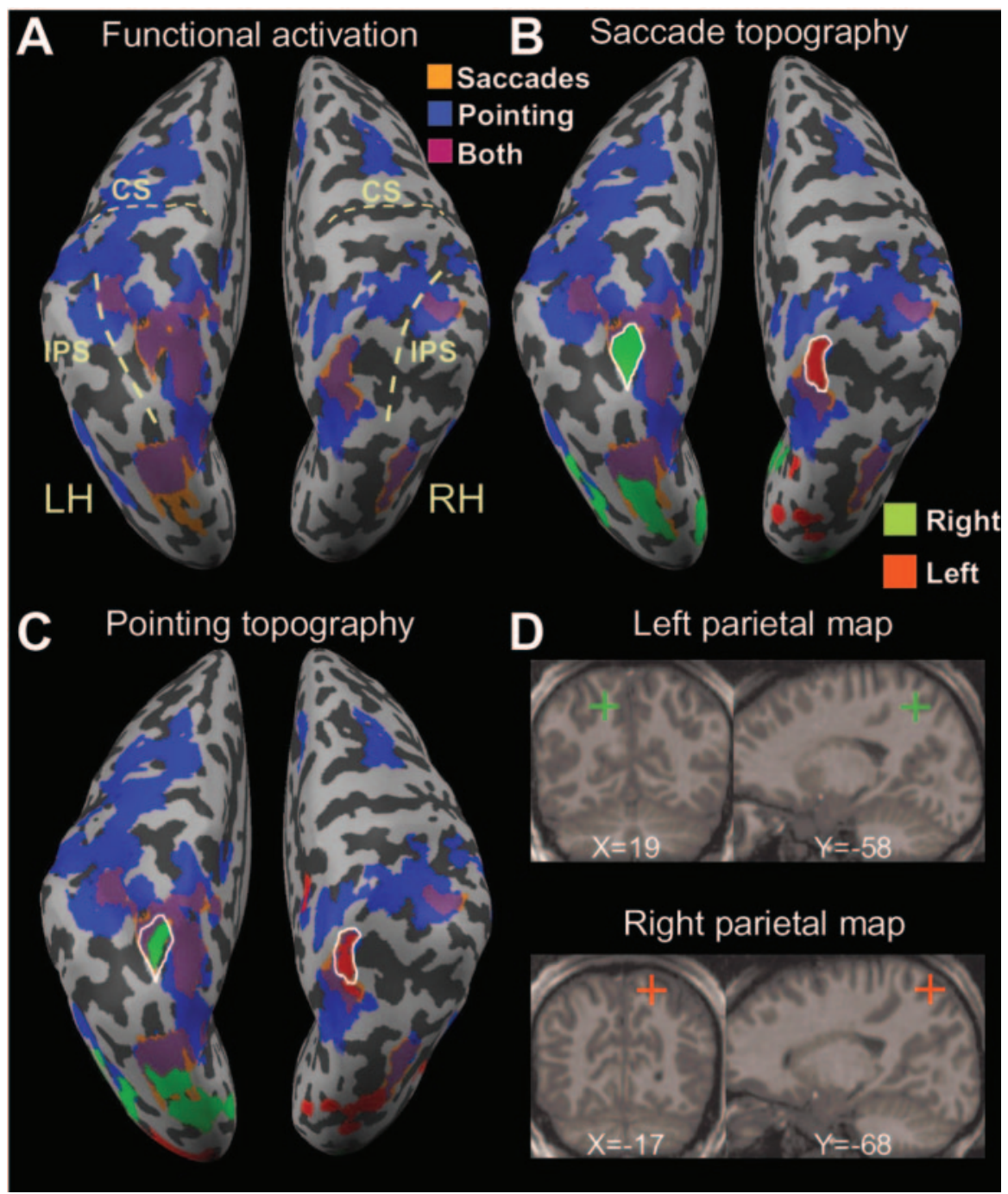

Figure 2. A subset of areas within the PPC shows topography for both saccade and pointing target locations. $A$, The regions showing higher activation for saccades or pointing movements than for fixation $\left(p<10^{-5}\right)$ in one subject, rendered onto an inflated representation of the cortical surface. Orange, Voxels activated during saccades. Blue, Voxels activated during pointing movements. Purple, Voxels activated during both saccades and pointing movements. CS, Central sulcus; IPS, intraparietal sulcus. $B$, Areas that show left-right topography for saccades $(p<0.001)$. C, The same regions show left-right topography for pointing movements $(p<0.001)$. D, The centers of the parietal maps marked in two slice views: a coronal and sagittal view. Green cross indicates left hemisphere; red cross indicates right hemisphere. Talairach coordinates were as follows (in mm): $x=-19, y=$ $-58, z=47$ (left) and $x=17, y=-68, z=55$ (right).

\section{Results}

In the first experiment, we used a delayed-movement task to identify human parietal areas implicated in saccades or pointing movements (Snyder et al., 1997; Batista et al., 1999; Sereno et al., 2001). In this task, illustrated in Fig. 1, a peripheral stimulus is flashed, and, after a $2.75 \mathrm{sec}$ delay, the subject makes either a saccade or a pointing movement toward its remembered location. As a first step in our analysis, we compared movement (either saccade or pointing) with no movement (fixation) to identify the cortical regions activated during saccades and pointing movements.

Figure $2 \mathrm{~A}$ shows the complete set of regions activated in both the saccade and pointing tasks of one subject, rendered onto an inflated representation of the cortical surface. The majority of the parietal regions that were activated during saccades were also activated during pointing movements (purple regions). Most of the remaining voxels were exclusively activated during pointing movements (blue regions), with fewer voxels activated exclusively by saccades (orange regions) (Connolly et al., 2000; DeSouza et al., 2000). We also observed activation in occipital and frontal areas, but here we focus on possible analogs of primate LIP and PRR in human PPC.

Which of these regions are topographically organized for saccades? To determine this at a simple level, we compared the PPC activation for leftward and rightward target locations. Red regions (Fig. $2 B$ ) indicate a stronger activation for remembered target locations to the left than to the right, whereas green voxels represent the opposite pattern. In the parietal cortex, a topographic zone was located at a medial branch of the intraparietal sulcus, in agreement with results by Sereno et al. (2001). As can be seen, the left hemisphere maps targets in the right hemifield, whereas the right hemisphere maps targets in the left hemifield. All subjects tested demonstrated an equivalently organized area in their PPC, mostly located within a small sulcus running medially from the intraparietal sulcus. Interestingly, the same set of PPC voxels also showed lateralized responses in the delayed-pointing task, as demonstrated by Figure $2 C$. Thus, the same PPC region showed topography for both saccades and pointing movements.

For anatomic reference, the center of this region of interest for this one subject is indicated for the left and right hemispheres in $D$ by the red and green crosses. Across subjects, the average Talairach coordinates (in millimeters) and their SDs of the peak parietal activation for saccades were $x=21(\mathrm{SD}=4), y=-62(\mathrm{SD}=9)$, $z=42(\mathrm{SD}=4)$ (right hemisphere) and $x=-19(\mathrm{SD}=5), y=-63(\mathrm{SD}=7), z=$ $46(\mathrm{SD}=5)$ (left hemisphere), consistent with but slightly more medial than the location reported by Sereno et al. (2001). For pointing, these coordinates were $x=22$ $(\mathrm{SD}=4), y=-64(\mathrm{SD}=9), z=44(\mathrm{SD}=4)$ (right) and $x=$ $-19(\mathrm{SD}=4), y=-62(\mathrm{SD}=7), z=46(\mathrm{SD}=6)$ (left). These were not significantly different from the location for saccades in any dimension (paired $t$ test; $p>0.05$ ).

The key question here is, does this area represent and update its information in a gaze-centered frame of reference? We tested this in experiment 2 using an event-related fMRI paradigm in which subjects produced intervening saccades between seeing a goal target and generating an eye or arm movement toward its remembered location (Fig. $1 B$ ) (see Materials and Methods). In all cases, the space-fixed goal target remained stable relative to the head and body. However, the remembered location of this target would have to be remapped during the intervening saccade to correctly code its new location in eye-fixed coordinates. As illustrated by Figure $3 A$, the paradigm consisted of four different conditions with regard to the location of the goal target relative to gaze direction before and after the first saccade; the goal target 
started and remained in the right visual hemifield (RR), or it started and remained in the left hemifield (LL), or it moved from the left into the right hemifield (LR), or vice versa (RL).

Figure $3 B$ shows the mean response of the six subjects in the left parietal region for each of the four conditions in the saccade-saccade task. As shown, after a brief presentation of the two targets, those for the initial saccade and the final goal, cortical activation builds up gradually during the first delay period, leading to a higher activity when the two targets were in the right (contralateral) hemifield than when they were in the left (ipsilateral) hemifield. The reverse is true for initial responses in the right parietal cortex (Fig. $3 C$ ). This reflects the topographical nature of the region, as identified in experiment 1 . The activity decays slightly during the first delay period, and then, when the first saccade occurs (i.e., $7.5 \mathrm{sec}$ after the start of the trial), cortical activation increases again, in all four conditions.

What happens after this saccade, in the second delay period? The activation of the region depends on the location of the remembered goal target relative to current gaze direction. For the left cortex (Fig. 3B), if the remembered goal shifted from the left hemifield (ipislateral) into the right (contralateral) hemifield (LR condition), a high sustained activation was observed in the second delay period. However, if it shifted from the right to left hemifield (RL condition), the level decreased. When the remembered goal target remained in the same hemifield after the first saccade, the activation was high if this location was contralateral (RR condition) and low if ipsilateral (LL condition). The right parietal region (Fig. 3C) showed a similar, but mirrored, pattern of activation.

Figure 3, D and E, shows a similar analysis for the four conditions in the saccadepoint task. Results in the saccade-point task were not as homogeneous as those in the saccade-saccade task. The presaccadic response is somewhat less clear compared with the saccade data in $B$ and $C$. However, the more important post-saccadic remapping response is just as robust and clear in the pointing data as for the saccade data. In other words, the saccade-point data showed the same gaze-centered remapping pattern, with symmetrically yoked activation between the left and right PPC. Together, these results suggest that, when the horizontal location of a remembered saccade or pointing goal reverses (leftright) with respect to gaze direction, these physical shifts are accompanied by dy-
A Before $1^{\text {st }}$ sac After $1^{\text {st }}$ sac
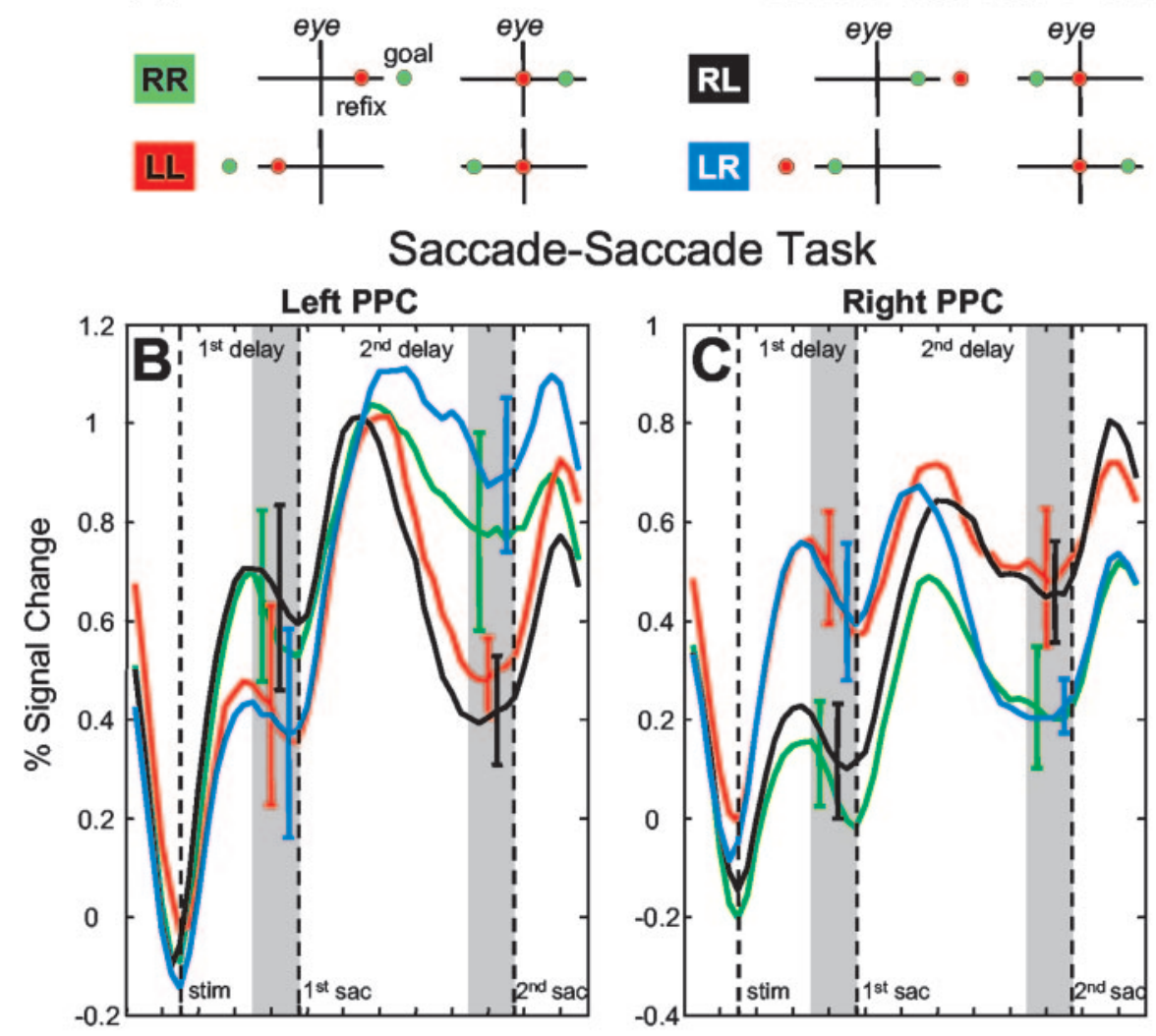

\section{Right PPC}

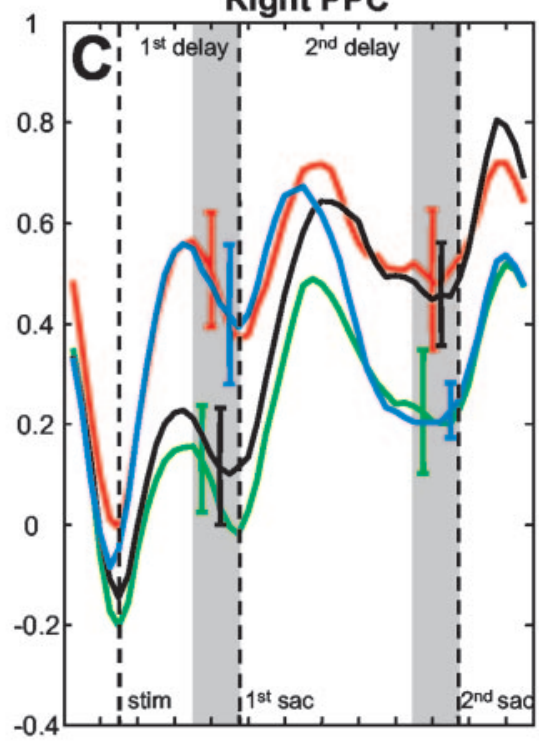

\section{Saccade-Point Task}
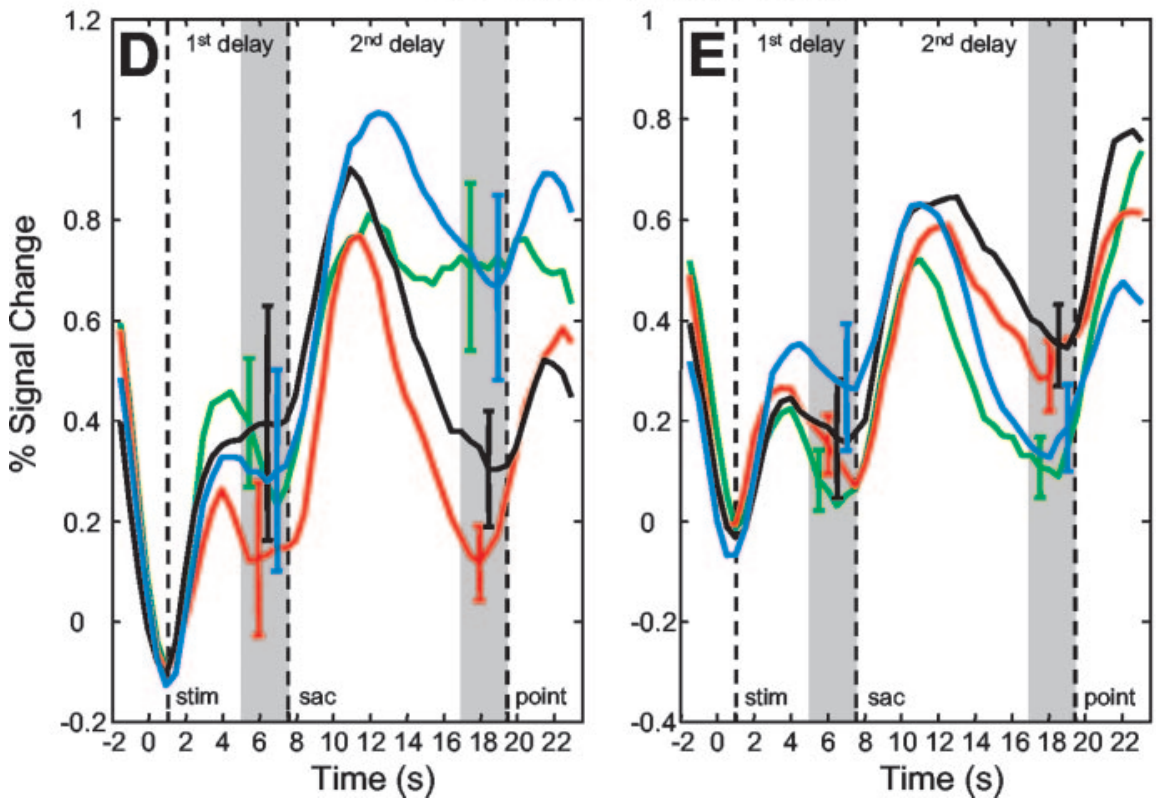

Figure 3. The remembered location of a target is transferred from one cerebral hemisphere to the other within the PPC. $A, R R$, $L L, R L$, and $L R$ signify four possible conditions of the intervening saccade paradigm; the first letter signifies initial location of the two targets ( $R$, right hemifield; $L$, left hemifield), and the second letter refers to the remapped location of the remembered goal location. Left $(B)$ and right ( $C$ parietal activation (mean \pm SE across 6 subjects) for each of the four conditions in the saccadesaccade task. SEs are plotted at the time point at which they were computed. All time courses are shifted to compensate for the fMRI hemodynamic lag. Dashed lines indicate presentation of stimuli, time of first saccade, and time of second saccade, respectively. Gray areas indicate the periods over which the differences between the LR and RL condition were taken. D, E, Saccade-Point Task, The remembered location of the goal target for hand pointing is transferred across cerebral hemispheres within the human PPC after an intervening saccade. Data in same format as $B$ and $C$. 


\section{A Saccade-Saccade Task B Saccade-Point Task}

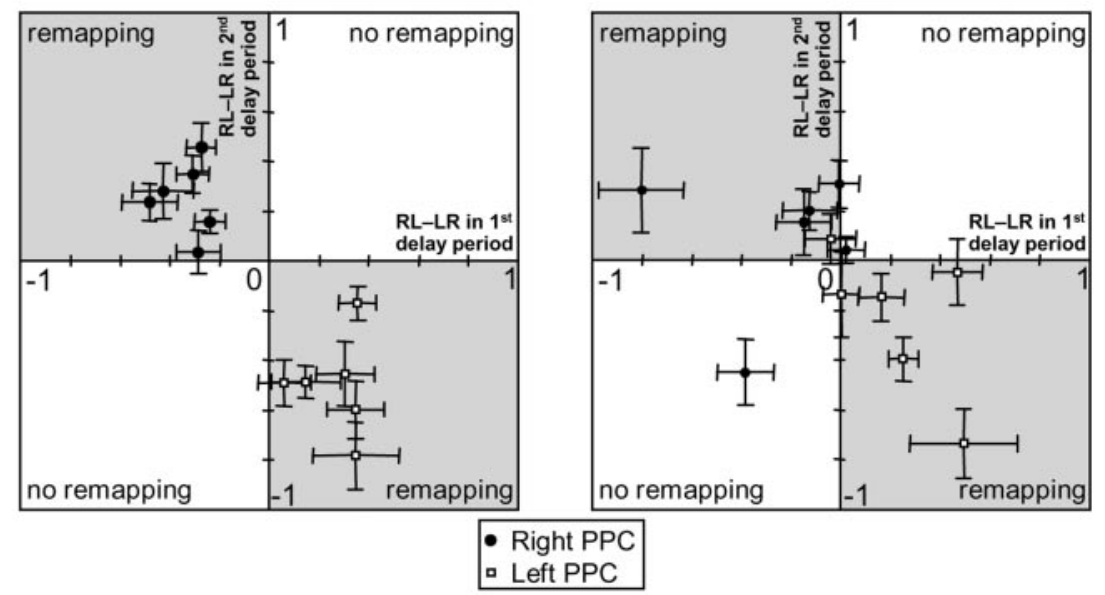

Figure 4. A comparison of activation before and after remapping in each subject. $A$, Goal is target for saccades. $B$, Goal is target for pointing movement. $x$-Axis, The difference ( $\pm S E$ ) in the average activation between the RL and LR conditions just before the first saccade. $y$-Axis, Same but after remapping. Filled circles, Right PPC; open squares, left PPC. Before the first saccade (first delay period) activation should be contralateral (i.e., $R L>L R$ for the left $P P C$, and $L R>R L$ for the right $P P C$ ). After the remapping (second delay period), activation should switch hemispheres (i.e., $L R>R L$ for left PPC, and RL $>$ LR for right PPC). Gaze-centered remapping requires right PPC data in the second quadrant and left PPC data in the fourth quadrant (gray zones).

namic shifts in cortical activity from one hemisphere to the other. This suggests that the parietal area identified here encodes and updates the remembered location of the saccade and pointing goals in eye-fixed coordinates.

To analyze these findings quantitatively, in Figure $4, A$ and $B$, we plotted the difference in activation between the RL condition and the LR condition after the first saccade (time period of 17$19.5 \mathrm{sec}$, second delay period) versus the difference before that saccade (time period of 5-7.5 sec, first delay period). These volumes are indicated schematically by the gray areas in Figure $3 B-E$. Gaze-centered updating requires that these differences have opposite sign. Accordingly, data from the right parietal cortex should be represented in the second quadrant, whereas left parietal data should be confined to the fourth quadrant (gray zones). A failure to update this information (or representation in a head-body fixed frame) would be indicated by data points in the opposite white zones. As Figure $4 A$ shows, the saccade-saccade data of all six subjects fell within the gaze-centered gray zones $(p<0.001 ; t$ test). Thus, all of our subjects encoded and updated visuospatial information for saccades in a gaze-centered reference frame. When we applied the same analysis to the saccade-point task data (Fig. $4 B$ ), nearly all of the data fell within the gaze-centered remapping zones ( $p<0.05 ; t$ test).

\section{Discussion}

Our results show that the topographic representation for goaldirected eye and pointing movements in human PPC is organized in gaze-centered, eye-fixed coordinates and is spatially updated across eye movements. This is consistent with the known physiology of the monkey PPC (Gnadt and Andersen, 1988; Duhamel et al., 1992a; Mazzoni et al., 1996; Batista et al., 1999) and with human psychophysics (McIntyre et al., 1997; Henriques et al., 1998; Medendorp and Crawford, 2002), which suggest an eyefixed coordinate system for representing reaching and pointing targets in both near and far space. This result does not contradict the idea that the same parietal regions might also be involved in implicitly transforming these gaze-centered signals into other reference frames (Andersen et al., 1985; Brotchie et al., 1995), with the ultimate goal of formulating commands in effectorcentered coordinates.

There is currently debate as to whether the posterior parietal cortex is more important for preparatory aspects of stimulus selection (Colby and Goldberg, 1999; Corbetta and Shulman, 2002; Yantis et al., 2002) or for response or action selection (Andersen et al., 1997; Snyder et al., 1997; Calton et al., 2002). For example, a recent study by Yantis et al. (2002) reported phasic, not tonic, changes in activity in the human PPC, related to attentional shifts in a visual recognition task. Our results show clear sustained responses (Fig. 3). This suggests that PPC responds in a more sustained manner for a task requiring an action to a remembered target location (like our task) compared with a task that only requires visual recognition.

The fact that the results are more apparent for the saccade-saccade task than for the saccade-point task (Fig. 4, compare $A, B)$ might indicate that our activation arises from a saccade area, which is only coactivated during arm movements (even when the saccade is suppressed) as part of some eye-hand coordination strategy (Snyder et al., 2000).

To distinguish whether our area specifically represents movements other than saccades, it would be useful to perform experiments that explicitly disassociate saccade planning from pointing movements (Snyder et al., 1997).

Our results could also mean that saccade and arm movement neurons are comingled in the same region with a greater preponderance of the former or a lesser need to sustain gaze-centered activity for pointing. Consistent with this, a recent report shows that neurons with arm-specific intention activity lie on both banks of the monkey intraprietal sulcus (Calton et al., 2002). Moreover, it is clear that damage to the human PPC results in deficits in programming both eye and arm movements (Duhamel et al. 1992b; Grea et al., 2002), and some of these reaching deficits are best explained in terms of a gaze-centered mechanism like that reported here (Khan et al., 2002). In summary, although our fMRI measurements cannot differentiate the proportion of cells involved in eye versus pointing movements, the simplest interpretation of our data are that human PPC is probably organized in a similar way as monkey PPC, i.e., there appears to be a regional overlap for eye and pointing movements.

Why would the human PPC operate in eye-fixed coordinates? Perhaps it is not surprising that the locations of targets for saccades are mapped relative to the current fixation point. At first glance, however, it might be unexpected that this also holds for pointing movements. It would seem more logical to code these in egocentric coordinates relative to the body. If, however, the function of the PPC is to select targets for action and the effectors to perform these actions (Snyder et al., 1997), it is critical that computations occur in a common coordinate frame. It would appear that this common coordinate frame is gaze centered (Batista et al., 1999). Ultimately, however, even the remapped input representations must be transferred into output coordinates. Indeed, this seems to be the case at the level of the frontal cortex (Grazi- 
ano et al., 2002). From primate studies, it is known that the PPC also possesses information on hand position (possibly also in eye-fixed coordinates; Buneo et al., 2002), eye position (Andersen et al., 1985), and head position (Brotchie et al., 1995). This places it in a unique position to begin the neural computations required for an accurate arm movement in motor coordinates, without the need for intervening high-level coordinate frames (Smith and Crawford, 2001). Together with the gaze-centered remapping mechanism described here, this provides the substrate for an economical, biologically compact visuomotor control system.

\section{References}

Andersen RA, Essick GK, Siegel RM (1985) Encoding of spatial location by posterior parietal neurons. Science 230:456-458.

Andersen RA, Snyder LH, Bradley DC, Xing J (1997) Multimodal representation of space in the posterior parietal cortex and its use in planning movements Annu Rev Neurosci 20:303-330.

Barash S, Bracewell RM, Fogassi L, Gnadt JW, Andersen RA (1991) Saccaderelated activity in the lateral intraparietal area. II. Spatial properties. J Neurophysiol 66:1109-1124.

Batista AP, Buneo CA, Snyder LH, Andersen RA (1999) Reach plans in eyecentered coordinates. Science 285:257-260.

Brotchie PR, Andersen RA, Snyder LH, Goodman SJ (1995) Head position signals used by parietal neurons to encode locations of visual stimuli. Nature 375:232-235.

Buneo CA, Jarvis MR, Batista AP, Andersen RA (2002) Direct visuomotor transformations for reaching. Nature 416:632-636.

Calton JL, Dickinson AR, Snyder LH (2002) Non-spatial, motor-specific activation in posterior parietal cortex. Nat Neurosci 5:580-588.

Colby CL, Goldberg ME (1999) Space and attention in parietal cortex. Annu Rev Neurosci 22:319-349.

Colby CL, Duhamel JR, Goldberg ME (1996) Visual, presaccadic, and cognitive activation of single neurons in monkey lateral intraparietal area. J Neurophysiol 76:2841-2852.

Connolly JD, Goodale MA, DeSouza JF, Menon RS, Vilis T (2000) A comparison of frontoparietal fMRI activation during anti-saccades and antipointing. J Neurophysiol $84: 1645-1655$.

Corbetta M, Shulman GL (2002) Control of goal-directed and stimulusdriven attention in the brain. Nat Rev Neurosci 3:201-215.

Corbetta M, Akbudak E, Conturo TE, Snyder AZ, Ollinger JM, Drury HA, Linenweber MR, Petersen SE, Raichle ME, Van Essen DC, Shulman GL (1998) A common network of functional areas for attention and eye movements. Neuron 21:761-773.

Culham JC, Kanwisher NG (2001) Neuroimaging of cognitive functions in human parietal cortex. Curr Opin Neurobiol 11:157-163.

DeSouza JF, Dukelow SP, Gati JS, Menon RS, Andersen RA, Vilis T (2000) Eye position signal modulates a human parietal pointing region during memory-guided movements. J Neurosci 20:5835-5840.

Duhamel JR, Colby CL, Goldberg ME (1992a) The updating of the representation of visual space in parietal cortex by intended eye movements. Science 255:90-92.

Duhamel JR, Goldberg ME, Fitzgibbon EJ, Sirigu A, Grafman J (1992b) Sac- cadic dysmetria in a patient with a right frontoparietal lesion. The importance of corollary discharge for accurate spatial behaviour. Brain 115:1387-1402.

Galletti C, Fattori P, Kutz DF, Battaglini PP (1997) Arm movement-related neurons in the visual area V6A of the macaque superior parietal lobule. Eur J Neurosci 9:410-413.

Gnadt JW, Andersen RA (1988) Memory related motor planning activity in posterior parietal cortex of macaque. Exp Brain Res 70:216-220.

Goodale MA, Milner AD (1992) Separate visual pathways for perception and action. Trends Neurosci 15:20-25.

Graziano MSA, Taylor CSR, Moore T (2002) Complex movements evoked by microstimulation of precentral cortex. Neuron 34:841-851.

Grea H, Pisella L, Rossetti Y, Desmurget M, Tilikete C, Grafton S, Prablanc C, Vighetto A (2002) A lesion of the posterior parietal cortex disrupts on-line adjustments during aiming movements. Neuropsychologia 40:2471-2480.

Hallett PE, Lightstone AD (1976) Saccadic eye movements to flashed targets. Vision Res 16:107-114.

Henriques DYP, Klier EM, Smith MA, Lowy D, Crawford JD (1998) Gazecentered remapping of remembered visual space in an open-loop pointing task. J Neurosci 18:1583-1594.

Jeannerod M, Arbid MA, Rizzolatti G, Sakata H (1995) Grasping objects: the cortical mechanisms of visuomotor transformation. Trends Neurosci 18:314-320.

Khan AZ, Crawford JD, Rossetti Y (2002) Updating of visual information across a delay in normals and optic ataxia patients. Soc Neurosci Abstr 28:57.1.

Mazzoni P, Bracewell RM, Barash S, Andersen RA (1996) Motor intention activity in the macaque's lateral intraparietal area. I. Dissociation of motor plan from sensory memory. J Neurophysiol 76:1439-1456.

McIntyre J, Stratta F, Lacquaniti F (1997) Viewer-centered frame of reference for pointing to memorized targets in three-dimensional space. J Neurophysiol 78:1601-1618.

Medendorp WP, Crawford JD (2002) Visuospatial updating of reaching targets in near and far space. NeuroReport 13:633-636.

Scherberger H, Goodale MA, Andersen RA (2003) Target selection for reaching and saccades share a similar behavioral reference frame in the macaque. J Neurophysiol 89:1456-1466.

Sereno MI, Pitzalis S, Martinez A (2001) Mapping of contralateral space in retinotopic coordinates by a parietal cortical area in humans. Science 294:1350-1354.

Smith MA, Crawford JD (2001) Self-organizing task modules and explicit coordinate systems in a neural network model for 3-D saccades. J Comput Neurosci 10:127-150.

Snyder LH, Batista AP, Andersen RA (1997) Coding of intention in the posterior parietal cortex. Nature 386:167-170.

Snyder LH, Grieve KL, Brotchie P, Andersen RA (1998) Separate body- and world-referenced representations of visual space in parietal cortex. Nature 394:887-891.

Snyder LH, Batista AP, Andersen RA (2000) Saccade-related activity in the parietal reach region. J Neurophysiol 83:1099-1102.

Yantis S, Schwarzbach J, Serences JT, Carlson RL, Steinmetz MA, Pekar JJ, Courtney SM (2002) Transient neural activity in human parietal cortex during spatial attention shifts. Nat Neurosci 5:995-1002. 